\title{
ON A QUESTION OF ARCHANGELSKIJ CONCERNING LINDELÖF SPACES WITH COUNTABLE PSEUDOCHARACTER
}

\author{
K. ALSTER
}

\begin{abstract}
We give a negative solution to Archangelskij's problem by showing that there exists a Lindelöf space with countable pseudocharacter which does not admit a continuous one-to-one mapping onto a first countable Hausdorff space.
\end{abstract}

The aim of this note is to construct, in the usual axioms of set theory, an example mentioned in the abstract (see [Ar, Hypotheses 5.4 and 5.5]). S. Shelah obtained, under some set-theoretical assumptions, a Lindelöf space of cardinality greater than $2^{\omega}$ with countable pseudocharacter (see [S and $\left.\mathbf{H J}\right]$ ). From the well-known Archangelskij theorem it follows that Shelah's space does not admit a continuous one-to-one mapping onto a first countable Hausdorff space.

Let us denote by $Q$ the set of rational numbers of the unit interval. The symbols $\omega$ and $\omega_{1}$ stand for the first infinite and first uncountable ordinal numbers respectively.

EXAMPLE. There is a Lindelöf space $X$ with countable pseudocharacter which does not admit a continuous one-to-one mapping onto a Hausdorff space satisfying the first axiom of countability.

Construction of $X$. There exists a family $\left\{A_{\alpha}: 1 \leqslant \alpha<\omega_{1}\right\}$ such that

(1) $A_{\alpha}$ is a countable set consisting of strictly increasing sequences of $Q$ of length $\alpha$ for $1 \leqslant \alpha<\omega_{1}$;

(2) if $\alpha<\beta<\omega_{1}$, then $p_{\alpha}\left(A_{\beta}\right)=A_{\alpha} ; p_{\alpha}$ stands for the projection onto the first $\alpha$ coordinates;

(3) if $a \in A_{\alpha}$ for $1 \leqslant \alpha<\omega_{1}$, then for every limit ordinal number $\beta<\alpha, a(\beta)=$ $\sup \{a(\lambda): \lambda<\beta\}$ and $\sup \{a(\lambda): \lambda<\alpha\}$ are rational numbers of $Q$;

(4) if $\alpha<\beta<\omega_{1}, a \in A_{\alpha+1}, r \in Q$ and $a(\alpha)<r$, then there exists $b \in A_{\beta+1}$ such that $p_{\alpha+1}(b)=a$ and $b(\beta)=r$ (see $[\mathbf{J}$, p. 91, the construction of the Aronszajn tree]).

Let us attach to $a \in A_{\alpha}$, for $1 \leqslant \alpha<\omega_{1}, x_{a} \in Q^{\omega_{1}}$ defined by

$$
x_{a}(\beta)= \begin{cases}a(\beta), & \text { if } \beta<\alpha, \\ \sup \{a(\lambda): \lambda<\alpha\}, & \text { if } \beta \geqslant \alpha .\end{cases}
$$

Let $X=\bigcup\left\{X_{\alpha}: 1 \leqslant \alpha<\omega_{1}\right\}$, where $X_{\alpha}=\left\{x_{a}: a \in A_{\alpha}\right\}$, be a subspace of $Q^{\omega_{1}}$. In [Al] it was proved that $Y \times X^{\omega}$ is Lindelöf provided that $Y$ is a hereditarily Lindelöf space. We shall sketch the proof of the Lindelöf property in $X$ for the sake of

Received by the editors February 29, 1984.

1980 Mathematics Subject Classification. Primary 54D20, 54A25.

(C)1985 American Mathematical Society $0002-9939 / 85 \$ 1.00+\$ .25$ per page 
completeness. Let $\mathscr{B}$ be a countable base of $Q$ and $\mathscr{V}$ an open covering of $X$. For $x \in X_{\alpha}, \alpha<\omega_{1}$, let

$\mathscr{A}(x)=\left\{B \in \mathscr{B}: x(\alpha) \in B\right.$ and there are $\alpha<\beta(x, B)<\omega_{1}$ and $V \in \mathscr{V}$

where

$$
\text { such that } \left.F(x, B, \beta(x, B))=\left(\prod_{\lambda<\omega_{1}} F_{\lambda}\right) \cap X \subset V\right\} \text {, }
$$

$$
F_{\lambda}= \begin{cases}\{x(\lambda)\}, & \text { if } \lambda \leqslant \alpha, \\ B, & \text { if } \lambda=\mathscr{B}(x, B), \\ Q, & \text { otherwise. }\end{cases}
$$

Since $X$ consists of increasing sequences, $\mathscr{A}(x) \neq \varnothing$ for every $x \in X$. Let

$$
\beta_{1}=\sup \left\{\beta(x, B): x \in X_{1} \text { and } B \in \mathscr{A}(x)\right\} \text {. }
$$

Since $X_{1}$ and $\mathscr{A}(x)$, for $x \in X_{1}$, are countable sets, $\beta_{1}<\omega_{1}$. If $\beta_{n}$ is defined, then let

$$
\beta_{n+1}=\sup \left\{\beta(x, B): x \in \bigcup\left\{X_{\lambda}: \lambda \leqslant \beta_{n}+1\right\} \text { and } B \in \mathscr{A}(x)\right\}
$$

and

$$
\beta=\sup \left\{\beta_{n}: n \in N\right\} \text {. }
$$

To finish the proof of the Lindelöf property of $X$ it is enough to show that

$$
X=\bigcup\left\{F(x, B, \beta(x, B)): x \in \bigcup\left\{X_{\lambda}: \lambda<\beta\right\} \text { and } B \in \mathscr{A}(x)\right\} \text {. }
$$

Let $x$ be an element of $X_{\lambda}$ for $\lambda \geqslant \beta$. Then $p_{\beta}(x)$ belongs to $A_{\beta}$. Let $x^{\prime}$ be a point of $X_{\beta}$ such that $p_{\beta}\left(x^{\prime}\right)=p_{\beta}(x)$. There exist $B \in \mathscr{B}, \alpha_{1}$ and $\alpha_{2}$ and $V \in \mathscr{V}$ such that $x^{\prime}(\beta) \in B, \alpha_{1}<\beta<\alpha_{2}$ and $F=\left(\prod_{\lambda<\omega_{1}} F_{\lambda}\right) \cap X \subset V$, where

$$
F_{\lambda}= \begin{cases}\left\{x^{\prime}(\lambda)\right\}, & \text { if } \lambda<\alpha_{1}, \\ B, & \text { if } \lambda=\alpha_{2}, \\ Q, & \text { otherwise. }\end{cases}
$$

Without loss of generality we can assume that $\sup \left\{x^{\prime}(\lambda): \lambda<\alpha_{1}\right\} \in B$. Let $v$ be an element of $X_{\alpha_{1}}$ such that $p_{\alpha_{1}}(v)=p_{\alpha_{1}}\left(x^{\prime}\right)$. Then $B \in \mathscr{A}(v)$ and $\beta(v, B)<\beta<\alpha_{2}$. It is easy to see that $x^{\prime} \in F(v, B, \beta(v, B))$. Since $p_{\beta}^{-1} p_{\beta}(F(v, B, \beta(v, B)))=$ $F(v, B, \beta(v, B))$ and $p_{\beta}\left(x^{\prime}\right)=p_{\beta}(x), x \in F(v, B, \beta(v, B))$. We conclude that $X$ is a Lindelöf space.

If $x \in X_{\alpha}, \alpha<\omega_{1}$, then $\left\{x^{\prime} \in X: p_{\alpha+2}\left(x^{\prime}\right)=p_{\alpha+2}(x)\right\}=\{x\}$ and $p_{\alpha+2}(X)$ is countable, so $\{x\}$ is a $G_{\delta}$-subset of $X$.

To finish the proof of the properties of $X$ it is enough to show that $X$ does not admit a weaker Hausdorff topology $\tau$ which satisfies the first axiom of countability. Suppose not and let $\tau$ be a weaker Hausdorff topology on $X$ satisfying the first axiom of countability. If $x \in X$ then there exists $\beta(x)<\omega_{1}$ such that for every open, in $\tau$, neighbourhood $U$ of $x$ there is a basic open neighbourhood $B(U)=$ $\left(\Pi_{\lambda<\omega_{1}} B_{\lambda}(U)\right) \cap X$ of $x$, with respect to the Tychonoff topology, such that $B_{\lambda}(U)=$ $Q$ for $\lambda \geqslant \beta(x)$ and $B(U) \subset U$. The existence of $\beta(x)$ is an immediate consequence of the fact that $\tau$ satisfies the first axiom of countability. Let $\beta_{1}=\sup \{\beta(x)$ : $\left.x \in X_{1}\right\}$. If $\beta_{n}$ is defined, then let

$$
\beta_{n+1}=\sup \left\{\beta(x): x \in \bigcup\left\{X_{\lambda}: \lambda \leqslant \beta_{n}+1\right\}\right\}
$$


and $\beta=\sup \left\{\beta_{n}: n \in N\right\}$. Notice that $\beta$ is a limit ordinal number less than $\omega_{1}$. Let $x_{1}$ and $x_{2}$ be points of $X_{\beta}$ and $X_{\beta+2}$, respectively, such that $p_{\beta+1}\left(x_{1}\right)=p_{\beta+1}\left(x_{2}\right)$ and $x_{1}(\beta+1)<x_{2}(\beta+1)$. To prove that $\tau$ is not a Hausdorff topology it is enough to show that if $U$ is an open neighbourhood of $x_{1}$, with respect to $\tau$, then there is a sequence $\left(y_{n}\right)_{n=1}^{\infty}$ of points of $U$ converging to $x_{2}$, with respect to the Tychonoff topology. Let $B \in \mathscr{B}$ and $\alpha_{1}, \alpha_{2}<\omega_{1}$ be such that $\beta_{1}<\alpha_{1}<\beta<\alpha_{2}, x_{1}(\lambda) \in B$ if $\lambda \geqslant \alpha_{1}$ and $F=\left(\prod_{\lambda<\omega_{1}} F_{\lambda}\right) \cap X \subset U$,where

$$
F_{\lambda}= \begin{cases}\left\{x_{1}(\lambda)\right\}, & \text { if } \lambda \leqslant \alpha_{1}, \\ B, & \text { if } \lambda=\alpha_{2} \\ Q, & \text { otherwise }\end{cases}
$$

Let $z_{n}$ be a point of $X_{\alpha_{n}+1}$, where $\alpha_{n}=\max \left\{\beta_{n}, \alpha_{1}\right\}$, such that $p_{\alpha_{n}+1}\left(z_{n}\right)=p_{\alpha_{n}+1}\left(x_{1}\right)$. Since $\beta$ is a limit ordinal number, $\alpha_{n}+1<\beta$. By the definition of $\beta\left(z_{n}\right)$, there exists a basic open neighbourhood $G\left(z_{n}\right)=\left(\prod_{\lambda<\omega_{1}} G_{\lambda}\left(z_{n}\right)\right) \cap X$ of $z_{n}$, with respect to Tychonoff topology, such that $G_{\lambda}\left(z_{n}\right)=Q$, if $\lambda \geqslant \beta\left(z_{n}\right)$ and $G\left(z_{n}\right) \subset U$. Notice that $\beta\left(z_{n}\right)<\beta$ for $n \in N$. Let $y_{n}$ be an element of $G\left(z_{n}\right) \cap X_{\beta+2}$ such that $p_{\alpha_{n}+1}\left(z_{n}\right)=p_{\alpha_{n}+1}\left(y_{n}\right)$ and $y_{n}(\lambda)=x_{2}(\lambda)$ for $\lambda \geqslant \beta$. The existence of $y_{n}$ is an easy consequence of $(4), \beta\left(z_{n}\right)<\beta, z_{n}(\lambda) \leqslant x_{1}(\lambda)$, for $\lambda<\omega_{1}, p_{\beta+1}\left(x_{1}\right)=p_{\beta+1}\left(x_{2}\right)$, and $x_{1}(\beta+1)<x_{2}(\beta+1)$. If $G=\left(\prod_{\lambda<\omega_{1}} G_{\lambda}\right) \cap X$ is a basic open neighbourhood of $x_{2}$, in the Tychonoff topology, $\alpha=\sup \left\{\lambda<\beta: G_{\lambda} \neq Q\right\}$ and $k$ is such that $\beta_{k}>\alpha$, then $y_{n} \in G$ provided that $n \geqslant k$, so we conclude that $\left(y_{n}\right)_{n=1}^{\infty}$ converges to $x_{2}$ in the Tychonoff topology.

REMARK. Let $\mathrm{Z}$ be a subspace of $I^{\omega_{1}}$, where $I$ stands for the unit interval, of all points of $I$ satisfying the following conditions:

(i) for every $\varepsilon>0$ and $z \in Z,\left\{\alpha<\omega_{1}: z(\alpha)>\varepsilon\right\}$ is finite;

(ii) for every $z \in Z,\left\{\alpha<\omega_{1}: z(\alpha)>0\right\}$ is an initial interval of $\omega_{1}$.

It is easy to see that $Z$ has countable pseudocharacter. In [C] it was proved that $Z$ has the Lindelöf property. Using our method one can show that $Z$ does not admit a continuous one-to-one mapping onto a first countable Hausdorff space.

\section{REFERENCES}

[A1] K. Alster, Some remarks on another Michael's problem concerning the Lindelöf property in the Cartesian products (in preparation).

[Ar] A. V. Archangelskij, On cardinal invariants, General Topology and its Relations to Modern Analysis and Algebra. III (Proc. Third Prague Topological Sympos. 1971), Academia, Prague, 1972, pp. 37-45.

[C] H. H. Corson, The weak topology of Banach space, Trans. Amer. Math. Soc. 101 (1961), 1-15.

[HJ] A. Hajnal and I. Juhász, Lindelöf spaces a la Shelah, Colloq. Math. Soc. János Bolyai 23 (1978), 555-567.

[J] T. J. Jech, Lectures in set theory with particular emphasis on the method of forcing, Lecture Notes in Math., vol. 217, Springer-Verlag, 1971.

[S] S. Shelah, Handwritten notes, 1978.

Institute of Mathematics of the Polish ACAdemy of Sciences, SNiadekich 8, 00-950 Warszawa, POLAND 